\title{
DE LA TRADICIÓN SÁFICA A LOS CÍRCULOS TRIBÁDICOS. LA BÚSQUEDA DE LAS IDENTIDADES LÉSBICAS DESDE UNA PERSPECTIVA HISTÓRICA (DE LA ANTIGÜEDAD CLÁSICA A LA EDAD MODERNA)
}

\author{
YOLANDA BETETA MARTÍN \\ Universidad Complutense de Madrid (UCM)
}

Recibido/12/03/2012

Aceptado/24/05/2012

\section{Resumen}

El artículo analiza la visibilidad y percepción social del lesbianismo desde una perspectiva histórica desde la Antigüedad Clásica hasta la Edad Moderna. Se inicia con un análisis de la estigmatización que ha padecido el estudio del lesbianismo en las Ciencias Sociales y a continuación analiza el tratamiento social, político e ideológico de las relaciones lésbicas desde la Antigüedad hasta el siglo XVII a través del estudio de las fuentes literarias, jurídicas e inquisitoriales. El estudio se centra en momentos históricos: la Grecia clásica, la Baja Edad Media y el siglo XVI debido a que la relevancia de la poetisa Safo, la Querella de las Mujeres y los ideales renacentistas contribuyen a matizar la percepción del lesbianismo. El objetivo del artículo es analizar la evolución de la percepción social del lesbianismo en las diferentes etapas históricas.

Palabras clave: Identidades lésbicas. Lesbianismo. Antropología de la Sexualidad. Historia de las Mujeres.

\section{Abstract}

The article discusses the visibility and social perception of lesbianism from the Classical Antiquity to the Modern Age. It begins with an analysis of the stigma that has suffered the study of lesbianism in the Social Sciences. Then the article analyzes the 
social, political and ideological treatment of lesbianism from Antiquity to the seventeenth century through the study of literary, inquisitorial legal sources. The study focuses on three historical moments: the Classical Greece, the Middle Ages and the sixteenth century because the relevance of the poet Sappho, the Complaint of Women and the Renaissance ideals influence the perception of lesbianism. The aim of this paper is to analyze the evolution of social perception of lesbianism in different historical periods.

Keywords: Lesbian identities. Lesbianism. Anthropology of Sexuality. Women's History. 
«Mujer que lo hace dos veces debe perder un miembro cada vez y a la tercera deber ser quemada».

El Libro de la Justicia y las Súplicas (1260).

\section{El estigma del lesbianismo en los estudios científicos. De la anomalía sexual a la resistencia patriarcal}

El estudio de la sexualidad y especialmente de las relaciones sexuales entre mujeres ha constituido un área marginal en las Ciencias Sociales. Hasta mediados del siglo XX la homosexualidad no formaba parte de los estudios históricos y antropológicos y menos aún las relaciones sexuales entre mujeres. Los trabajos sobre relaciones lésbicas sufrieron la autocensura en la mayor parte del siglo XX pese a que existían investigaciones antropológicas sobre relaciones sexuales entre mujeres desde al menos los años cuarenta. El estigma académico asociado a la investigación de todas aquellas relaciones que cuestionaran la primacía de la heterosexualidad como engranaje básico de la sociedad está en la base de la censura autoimpuesta por las/os investigadoras/ es. Clásicos de la antropología como Evans-Pritchard y Rudolf Van Lier padecieron la desconfianza que las relaciones sexuales entre mujeres despertaban en el ámbito científico. Evans-Pritchard publicó su trabajo sobre la «inversión sexual de los azandes» en el año $1970^{1}$, cuarenta años después de realizar el trabajo de campo, y Van Lier, que se interesó por las relaciones mati entre las mujeres creole de Paramaribo en el año 1947, no publicó sus conclusiones hasta la década de los ochenta denominando a esas relaciones «tríbadas tropicales» ${ }^{2}$.

Otras/os autoras/es como Bronislaw Malinowski, Ruth Benedict y Margaret Mead sí publicaron trabajos de campo en los que subrayan la necesidad de incorporar la sexualidad en los estudios de género pero sin dedicar una especial atención a las relaciones entre mujeres. El tabú de la homosexualidad

1. EvANS-PRITCHAR, Edward Evans. «Sexual inversión among the Azandes». American Antropologist 72/6 (1970), pp. 1428-1434.

2. VAN LIER, Rudolf. Tropische Tribaden: Een Verhandeling over Homoseksualiteit en Homoseksuele Vrouwen in Suriname. Frankfurt, Foris Publications, 1986. 
lésbica pervivió en el mundo académico hasta los años ochenta como señala la decisión de la antropóloga Saskia Wieringa de publicar sus estudios sobre las relaciones lésbicas en Yakarta bajo pseudónimo ${ }^{3}$. No será hasta finales de los ochenta cuando el movimiento queer, las revisiones del construccionismo social, la teoría perfomativa del sexo de Judith Butler ${ }^{4}$ y las teorías feministas faciliten la entrada de los estudios sobre relaciones sexuales entre mujeres en los Estudios de Género y Estudios Feministas.

Uno de los argumentos que se han esgrimido para justificar el escaso interés de la comunidad científica por la sexualidad lésbica es la invisibilidad social de dichas relaciones. Sin embargo, como señala Evelyn Blackwood, esa invisibilidad en muchas ocasiones respondía a las limitaciones de los observadores y no a la manera en que las mujeres sentían, vivían y percibían su propia sexualidad $^{5}$. La interiorización de la heterosexualidad como el mecanismo básico universal que regula las relaciones sociales, ha limitado la forma en que las Ciencias Sociales han estudiado las relaciones homosexuales y en especial las relaciones de carácter lésbico. Mientras que la historiografía ha aceptado la publicación de estudios sobre la homosexualidad masculina en las sociedades clásicas, son muy pocas las investigaciones publicadas sobre las relaciones lésbicas en las distintas etapas históricas. Esta ausencia no indica que no existieran tales relaciones sino que incluso dentro de las investigaciones históricas y antropológicas sobre la homosexualidad existe una discriminación patente de los estudios lésbicos.

La discriminación de los estudios lésbicos no sólo se debe a la perspectiva androcéntrica y patriarcal que ha caracterizado a las disciplinas científicas sino que, además, se incrementa con la percepción del lesbianismo como una anomalía. La interpretación del lesbianismo como una anomalía o desviación determinó los estudios históricos y antropológicos hasta décadas recientes y constituyó el núcleo conceptual de las teorías deslegitimadoras del lesbianismo. Una de las teorías más extendidas era la que consideraba que

3. WieRINGA, Saskia.»Feminist Anthropology since the Mid-Seventies: From Monocausality to Diversity, a personal view», en M. Krueger (ed.): Was Heisst Hier Eigentlich Feministisch?, Frankfurt, Donat, 1993.

4. Butler en su obra El Género en disputa. Feminismo y la subversión de la identidad (1990) sobrepasa el construccionismo social de la categoría género y afirma que el sexo y la sexualidad lejos de ser fenómenos naturales o biológicos son, como el género, construcciones sociales.

5. BLACKWOOD, Evelyn. «Breaking the mirror: The construction of Lesbianism and the Anthropological Discourse on Homosexuality» en Evelyn Blackwood (ed.): The many faces of Homosexuality: Anthropological approaches to Homosexual Behavior, Harrington Park Press, 1986. 
las relaciones lésbicas sólo tienen cabida en los grupos sociales en los que el número de hombres es notablemente inferior al de mujeres. Tradicionalmente se ha asumido que las prácticas sexuales lésbicas son inasumibles si existe el suficiente número de hombres para satisfacer las necesidades sexuales tal y como propuso Raymond Firth respecto a las relaciones entre los tikopia de Melanesia ${ }^{6}$. La creencia de que las relaciones lésbicas sólo surgen en grupos en los que existe «privación heterosexual», ya sea debido a la inferioridad numérica de los hombres o a una segregación de sexos, se difundió con fuerza en muchos círculos intelectuales muy influenciados por los relatos de viajeros que a principios del siglo XX informaron sobre la existencia de harenes u hogares poligínicos en Oriente.

La percepción de los harenes orientales como espacios con una fuerte carga sexual entre mujeres constituye la base de la teoría del lesbianismo como una privación heterosexual. Los análisis antropológicos que sustentaron esta teoría padecen una fuerte connotación androcéntrica y eurocéntrica motivada por las proyecciones de los tópicos y fantasías sexuales de los investigadores ante los míticos harenes orientales ${ }^{7}$. Un ejemplo clásico de la teoría de la privación como explicación del lesbianismo aplicada a los grupos políginicos orientales es la que propusieron los historiadores Carey y Houben sobre el estudio previo realizado por Winter en Surakarta (Java oriental) en $1902^{8}$. Ambos historiadores proyectaron sus prejuicios sobre las relaciones lésbicas alegando que las mujeres de los harenes no disfrutaban de las relaciones lésbicas y que su aceptación se debía exclusivamente a la ausencia de hombres.

El rechazo visceral a las relaciones lésbicas por parte de la historiografía antropológica tradicional se acrecentó con la ocultación de tales relaciones por parte de los investigadores ${ }^{9}$. Además, en muchos casos, las relaciones lésbicas fueron eliminadas o desvirtuadas tras las transformaciones estructurales impulsadas por las culturas y religiones occidentales de base patriarcal sobre sus territorios coloniales. Un ejemplo de las consecuencias coloniales sobre la percepción social del lesbianismo es la deslegitimación que se realizó sobre las mujeres guerreras fon de Benín bajo las acusaciones de inmoralidad

6. FIRTH, Raymond. We, the Tikopia, London. American Book, 1936.

7. Murray, Stephen \& RosCoE, Will. Islamic Homosexualities: Culture, History and Literature. New York, University Press, 1997.

8. CAREY, Peter \& HoubEN, Vincent. «Spirited Srikandhis and Sly Sumabadras: The social, political and economic roles of women at the Central Javanese Courts in the XVIII and the early XIXth Centuries» en Elsbeth Locher-Scholten \& Anke Niehof (eds.): Indonesian women in Focus, Dordrecht, Foris Publications, 1980.

9. POVINELLI, Elizabeth. «Sexual savages/sexual sovereignty: Australian colonial texts and the postcolonial politics of nationalism» en Diacritics 24/2-3 (1994), pp. 122-150. 
y desviación de la norma heterosexual. Hoy las mujeres guerreras fon sólo permanecen en la memoria colectiva como amazonas a las que no les estaba permitido casarse ni tener descendencia y que tenían hetaíras a su disposición para satisfacerlas sexualmente ${ }^{10}$.

Los primeros estudios históricos y antropológicos sobre lesbianismo surgen, por tanto, a raíz de los trabajos de campo realizados en sociedades extraeuropeas que bajo una perspectiva eurocéntrica y colonial califican las relaciones sexuales entre mujeres como fenómenos exóticos y/o primitivos que vulneran la norma heterosexual. El rechazo que el estudio de la sexualidad lésbica despierta en el ámbito académico motiva que su análisis no sólo se incorpore tarde a las disciplinas históricas y antropológicas sino que, además, se perfile como un fenómeno aislado, primitivo y precolonial que contribuye a invisibilizar las relaciones entre mujeres en las sociedades modernas.

A mediados del siglo XX el surgimiento de la antropología de la sexualidad convierte las prácticas e identidades sexuales en objeto de estudio. Las investigaciones focalizan la atención fundamentalmente en las relaciones homosexuales masculinas argumentando que el lesbianismo era menos visible socialmente y menos desarrollado transculturalmente ${ }^{11}$.Una aparente invisibilidad que, no obstante, no impidió la proliferación de diversas teorías sobre la sexualidad lésbica que en muchas ocasiones se perfilaba como un imagen especular de la homosexualidad masculina al asumir que una práctica sexual estructuralmente análoga es similar en hombres y mujeres.

No es hasta la década de los años ochenta cuando la antropología de la sexualidad insiste en la necesidad de distinguir entre las prácticas homosexuales masculinas y femeninas como afirma Adrianne Rich al indicar que cualquier teoría sobre el lesbianismo basada en los principios de las relaciones masculinas nace debilitada y sólo contribuye a deformar los estudios transgénericos $^{12}$. Evelyn Blackwood se posiciona en el mismo sentido y argumenta que en la medida en que la sexualidad está conformada por ideologías y jerarquías de género que imponen marcos normativos diferentes en hombres y mujeres los comportamientos, roles sexuales, significados y deseos deben ser necesariamente distintos en las relaciones homosexuales y en las relaciones

10. KARSCH-HAACK, Ferdinand. Das Gleichgeschlechtliche leven der Naturvölker. München, Reinhardt, 1911.

11. GEBHARD, Paul. «Human sexual behavior. A summary statement» en Donald Marshall \& Robert C. Suggs (eds.): Human sexual behaviour. Variations in the Etnographic Spectrum, New York, Basic Books, pp. 206-217.

12. Rich, Adriane. «Compulsory Heterosexuality and lesbian existence» en Signs 5 (4), pp. 631-660. 
lésbicas ${ }^{13}$. El estudio del lesbianismo en las últimas décadas se desarrolla bajo estas perspectivas constructivistas que subrayan el carácter sociocultural y no biologicista de la sexualidad y desde la consideración de que el lesbianismo y las identidades lésbicas no son homólogas a la homosexualidad masculina.

La interiorización de que el lesbianismo no es una simple práctica sexual entre mujeres sino que adquiere un significado complejo dentro de los distintos contextos culturales que lo enmarcan permite impulsar y dinamizar los debates antropológicos bajo conceptos tan atrayentes como «heterosexualidad obligatoria» o «resistencia lésbica». La noción de «heterosexualidad obligatoria» impulsada por Adrianne Rich ha permanecido como uno de los pilares de las teorías lésbicas bajo la afirmación de que la heterosexualidad es un mecanismo sociocultural impuesto por el patriarcado para regular la sexualidad y perpetuar la dominación masculina ${ }^{14}$. Han surgido voces discrepantes como la de Jacqueline Zita que critican la excesiva rigidez del concepto porque constriñe la sexualidad femenina ${ }^{15}$. Sin embargo, la universalización de la heterosexualidad ha perfilado el lesbianismo como una «transgresión sexual»o «resistencia lésbica» a las imposiciones normativas del patriarcado que ha calado profundamente en la cultura lésbica.

Autoras como Adrianne Rich y Cherryl Clarke ofrecen una visión netamente transgresora y atrayente del lesbianismo pero lo cosifican en la medida en que lo definen exclusivamente como un acto de resistencia ${ }^{16}$. Es cierto que la heterosexualidad constituye el mecanismo básico que rige las relaciones sociales y sexuales de la mayoría de las sociedades y, por tanto, mantiene un carácter obligatorio y normativo. Los estudios sobre las prácticas lésbicas entre las mujeres de las hermandades chinas refuerzan el carácter transgresor de tales prácticas porque socialmente se interpretan como una ruptura del matrimonio heterosexual a favor de una vida independiente basada en los vínculos afectivos con otras mujeres ${ }^{17}$. No obstante, entender el lesbianismo como un acto de resistencia también constriñe la sexualidad femenina porque se concibe exclusivamente como una respuesta de negación o desobediencia a la heterosexualidad diluyendo la pluralidad de las prácticas sexuales y no da respuesta al significado que tales prácticas tienen en las sociedades que

13. BLACKWOOD, Evelyn. Ibidem.

14. RicH, Adrianne. Ibidem.

15. ZITA, Jacqueline. «Historical amnesia and the lesbian continuum», en Signs: Journal of women in culture and society, 7 (1), pp. 172-187.

16. Clarke, Cherryl. Lesbianism: an act of resistance. London, Persephone Press, 1981

17. SANKAR, Andrea. «Sisters and brothers, lovers and enemies» en en Evelyn Blackwood (ed.): The many faces of Homosexuality: Anthropological approaches to Homosexual Behavior, Harrington Park Press, 1986. 
legitiman el lesbianismo. Los estudios antropológicos sobre las relaciones lésbicas entre las mummies y babies de Lesotho (mujeres adultas y chicas adolescentes respectivamente) no constituyen actos de resistencia sino relaciones afectivas con un carácter iniciático en el que las adolescentes se adentran en el mundo de las relaciones sociales y afectivas de la vida adulta ${ }^{18}$.

La existencia de relaciones lésbicas socialmente reconocidas cuestiona el carácter exclusivamente transgresor del lesbianismo. Las prácticas lésbicas no se pueden limitar a un acto de transgresión o resistencia, en la medida en que están arraigadas en las relaciones sociales de muchas culturas, y adoptan un carácter afectivo y amoroso que va más allá del cuestionamiento de la heterosexualidad patriarcal. La antropología de la sexualidad ha pasado de analizar el lesbianismo como una anomalía a perfilarlo como un acto de resistencia. Sin embargo, las profundas imbricaciones socioculturales entre fenómenos como la transversalidad de género, la construcción de las identidades y los significados culturales abrieron nuevas líneas de investigación sobre el lesbianismo que inciden no tanto en el carácter sexual como en su carácter identitario.

\section{De la tradición sáfica a los círculos tribádicos. La construcción de las identidades lésbicas desde una perspectiva histórica}

La construcción de las identidades lésbicas es un complejo proceso en el que se imbrican diversos fenómenos de naturaleza sociocultural que, en mayor o menor medida, derivan de las identidades de género, núcleo primario de la construcción de las identidades. En dicha construcción se pueden distinguir dos conceptos clave sobre los que se articula todo el proceso identitario: los roles de género, impuestos socioculturalmente en función del sexo de los individuos, y la autoidentidad o percepción que los individuos tienen de sí mismos y que, por lo tanto, tiene un carácter netamente subjetivo e individual. La coexistencia entre los roles de género y la propia subjetividad que cada individuo tiene de sí mismo convierte la construcción de las identidades en un proceso dinámico y dialéctico en el que no siempre se produce una connivencia entre ambos fenómenos ${ }^{19}$. En el caso de las identidades lésbicas la ruptura entre los roles de género y la autoidentidad emana de la transgresión de la normativa heterosexual. Frente a la imposición de unas relaciones

18. GAY, Judith. Mummies and babies and friends and lovers in Lesotho. Harrington Park Press, 1986.

19. LAGARDE, Marcela. Género y feminismo: derechos humanos y democracia. Madrid, Horas y Horas, 2000. 
sexuales entre personas de distinto sexo surge una autoidentidad lésbica que vulnera las construcciones sociales dadas y que gira en torno a la sexualidad como núcleo definitorio del sujeto dentro de una red más amplia de experiencias y significados vitales.

La construcción de las identidades es un aspecto central en los estudios contemporáneos sobre el lesbianismo y, desde esta premisa, las investigaciones se han centrado en el análisis histórico-cultural de los comportamientos lésbicos a partir de las regulaciones normativas que sobre la sexualidad impone cada cultura y periodo histórico. El tema central que se aborda a continuación es la percepción social del lesbianismo en Occidente a lo largo de los diversos periodos históricos, atendiendo a las normativas sexuales y a la visibilidad de las prácticas y comportamientos lésbicos vigentes desde la Antigüedad Clásica a la Edad Moderna. Este breve recorrido por la historia del lesbianismo ofrece una visión global de la construcción de las identidades lésbicas desde una perspectiva histórica. Con ello se pretende abrir nuevas vías de investigación en un campo de estudio absolutamente marginal dentro de la Historia y que contrasta con el creciente interés mostrado por la Antropología.

La Historia es una disciplina que tradicionalmente ha adoptado un posicionamiento androcéntrico, que no sólo ha minusvalorado la importancia de las mujeres en el devenir histórico sino que, además, ha deslegitimado las actitudes y roles sociales que no se adecuaban a las modelos normativos de feminidad diseñados por la mentalidad patriarcal. En este sentido, no resulta sorprendente que la historiografía haya silenciado las relaciones lésbicas a lo largo de la Historia. Una parcialidad historiográfica que se ha visto acrecentada por la escasez de fuentes históricas e iconográficas referidas al lesbianismo y por los prejuicios de los propios investigadores que han proyectado sus creencias y dogmas en el estudio del pasado histórico.

El interés de la sociedad europea del siglo XVIII por la Antigüedad Clásica y la consiguiente exaltación de las artes grecorromanas como paradigma de los ideales modernos originan una idealización de la Antigüedad basada en unos principios patriarcales, eurocéntricos e ilustrados. A partir de los principios ilustrados y coloniales los estudios históricos interpretaron la sociedad grecorromana como un ente colectivo masculino en el que las mujeres no trascienden como sujetos históricos. Si la Historia de las Mujeres no despertó el interés de los estudios neoclásicos menos aún lo hizo el lesbianismo pese a que sí se publicaron estudios referidos a la homosexualidad masculina aunque de carácter marginal.

Pese a que existen referencias de relaciones sexuales y amorosas entre hombres en la literatura, el arte y la filosofía como resultado del carácter 
homosocial de la mentalidad clásica, no se han detectado registros históricos sobre relaciones lésbicas. Esto no se debe a la ausencia de relaciones lésbicas sino al hecho de que las relaciones homosexuales entre mujeres no fueron integradas en el sistema social, es decir, no adoptan una connotación iniciática, social y/o militar socialmente reconocida.

La literatura griega ofrece algunas alusiones de relaciones afectivas y sexuales entre mujeres que son descritas de forma análoga a las relaciones homosexuales masculinas. Una de las primeras referencias a relaciones lésbicas en la literatura griega es la expresada por el poeta Alcmán de Esparta ${ }^{20}$ que empleó el término aitis como la forma femenina de aites, que era la denominación con la que se designaba al individuo más joven de una relación homosexual $^{21}$. En el diálogo platónico El banquete ${ }^{22}$, Aristófanes ${ }^{23}$ - uno de los comensales del banquete que da nombre a la obra - menciona a mujeres que mantienen relaciones afectivas y sexuales con otras mujeres pero emplea el término trepesthai ( «estar centrado sobre») en lugar de eros, que era la palabra empleada para designar las relaciones eróticas heterosexuales y homosexuales masculinas ${ }^{24}$. Esta diferencia etimológica indica que las relaciones lésbicas eran percibidas como fenómenos distintos a las relaciones homoeróticas mantenidas entre los hombres aunque no de ello no se puede determinar el grado de aceptación o permisividad social de las relaciones entre mujeres.

El valor de la intervención de Aristófanes en El Banquete respecto al tema que nos ocupa radica no sólo en las significaciones etimológicas sino en su visión mítica del origen de la diferenciación sexual. Describe que la tierra estuvo habitada por personas esféricas con dos caras, cuatro piernas, cuatro brazos que pertenecían a tres sexos distintos: el masculino, descendiente del sol; el femenino, descendiente de la tierra y el andrógino, descendiente de la luna que poseía órganos reproductivos masculinos y femeninos. La arrogancia de estos seres provocó la ira de Zeus que para someterlos dividió sus cuerpos con su rayo convirtiéndolos en seres incompletos y condenados a anhelar la

20. Alcmán de Esparta (s. VII a.C). Poeta lírico que forma parte del Canon que integra a los nueve poetas más reputados según los dictados de la Escuela de Alejandría.

21. BREMmER, Jan (ed.). From Sappho to de Sade: Moments in the History of Sexuality. Routledge, 1989.

22. El banquete es un diálogo, en el que Platón presenta a varias personalidades relevantes de Atenas en tiempos de Sócrates compartiendo mesa y discutiendo sobre el amor. Entre ellos, Aristófanes relata un mito de la creación y la diferenciación sexual.

23. Aristófanes (444 a.C). Dramaturgo griego y principal exponente del género de comedia. Sus obras recogen intensas discusiones ideológicas, políticas, filosóficas, económicas y literarias en la Atenas del siglo $\mathrm{V}$ a.C.

24. AldRICH, Robert (ed.). Gay Life and Culture: A World History, Thames \& Hudson, 2006.

Feminismo/s 19, junio 2012, pp. 29-49 
unión con su mitad perdida. Del mito se desprende que los dos primeros sexos buscan su otra mitad corporal correspondiente a su propio sexo mientras que los seres lunares o andróginos buscan su otra mitad que corresponde necesariamente al otro sexo. Si la unión de las dos mitades se realiza a través de las relaciones sexuales, entonces las relaciones homosexuales, lésbicas y heterosexuales correspondientes a cada uno de los tres sexos están reconocidas en el mismo grado de igualdad. Es decir, la lógica griega admitía que las relaciones lésbicas mantienen el mismo grado de reconocimiento que las relaciones homosexuales y, a su vez, que ambas relaciones tiene la misma libertad natural que las relaciones heterosexuales.

Aunque la filosofía reconocía las relaciones homosexuales, lésbicas y heterosexuales en términos de igualdad, las relaciones sexuales entre mujeres no se percibían socialmente al mismo nivel que las relaciones entre hombres. Es significativo que la mitología griega no ofrezca en sus mitos relaciones entre diosas de forma análoga a los mitos que consagraron las relaciones homosexuales entre deidades masculinas. Además, no han trascendido en el imaginario colectivo amistades nobles entre mujeres de relevancia social, política e histórica similares, por ejemplo, a las de Harmodio y Aristogitón o Cratino y Aristodemo.

Historiográficamente se ha destacado la figura de Safo y las poetisas de Lesbos como el paradigma simbólico de la pasión entre mujeres. Sin embargo, no se puede establecer una comparativa entre las representaciones literarias y la realidad social. Es cierto que la poesía sáfica concede a las mujeres y sus pasiones un lugar eminente en la literatura clásica, pero aún en el caso de que las relaciones lésbicas fueran socialmente reconocidas en Eolia dicho reconocimiento no hundió sus raíces en el conjunto de Grecia.

La sociedad helénica estaba cimentada sobre valores profundamente androcéntricos, basados en los conceptos de fatría masculina y violencia bélica, por lo que no resulta sorprendente que las relaciones homosexuales entre hombres fueran socialmente reconocidas bajo una perspectiva social, militar, iniciática e ideológica. La relegación de las mujeres en la esfera doméstica y la escasez de referencias históricas sobre el lesbianismo revela que en la Antigüedad Clásica las relaciones sexuales entre mujeres se percibían más como una anomalía o una excentricidad, que contrasta con las lecturas sáficas y con los principios filosóficos derivados del Aristófanes platónico, que como una relación sexual socialmente reconocida.

La aparición y expansión del cristianismo constituye un punto de inflexión respecto a la consideración social del lesbianismo. El discurso patrístico sanciona cualquier relación sexual que se produzca fuera del matrimonio 
canónico independientemente de la naturaleza de tal relación. No obstante, los autos inquisitoriales relacionados con casos de sodomía evidencian que la reprobación de las relaciones homosexuales era claramente superior a cualquier otro tipo de relación sexual extramarital.

El estudio del lesbianismo en la Edad Media presenta numerosas dificultades ya que la mayoría de las referencias documentales sobre la homosexualidad se refieren a relaciones entre hombres donde, además, se entremezclan las acusaciones de homosexualidad, sodomía y herejía. Es difícil encontrar referencias históricas de relaciones sexuales entre mujeres aunque se registran informaciones veladas en los documentos jurídicos, archivos eclesiásticos y fuentes literarias. La mayoría las referencias se inspira directamente en los discursos de los Padres de la Iglesia. San Ambrosio calificó en el siglo IV el deseo de las mujeres entre sí de actos lujuriosos, San Juan Crisóstomo los calificó de vergonzosos y, siglos más tarde, San Anselmo y Pedro Abelardo se referirían a la relaciones entre mujeres como un atentado contra la naturaleza cristiana. Más trascendencia tuvieron las afirmaciones de Santo Tomás que definió «la cópula entre hembra y hembra» como un de los vicios contra natura. Esta visión de Santo Tomás constituyó la base doctrinal sobre que se condena penal y teológicamente el lesbianismo bajo la acusación del pecado de la lujuria.

La primera iniciativa legislativa que condena el lesbianismo explícitamente como un fenómeno distinto a la homosexualidad masculina se recoge en el código Livres de jostice et de plet ${ }^{25}$ promulgado en Orleans en el año 1260. El Livres recoge diversos aspectos legales referidos a lo que define como «delitos sexuales», entre los que se registra la homosexualidad. En el caso de la homosexualidad masculina, se establecen tres sanciones distintas dependiendo del número de delitos sexuales que acumule el acusado. Tras el primer delito el sodomita sería castrado, tras el segundo sería desmembrado y después de la tercera acusación sería quemado en la hoguera. En el caso de las mujeres acusadas de mantener relaciones sexuales con otras mujeres, las dos primeras acusaciones conllevan la desmembración corporal y la tercera acusación conlleva el castigo en la hoguera. En ambos casos la ejecución del acusado/a conlleva la inmediata confiscación de sus bienes por parte de la corona ${ }^{26}$.

25. El Libro de las justicias y las súplicas es un tratado jurídico elaborado por la denominada Escuela Comentarista que durante los siglos XI y XII sentó las bases de la legislación altomedieval a partir del corpus jurídico romano. El Libro se inspira en el derecho canónico, sobre toso los Decretales de Gregorio IX, el derecho romano y el derecho consuetudinario de la dinastía Capeta.

26. Rapetti, Pierre-Nicolas Rapetti \& Chabaille Polycarpe (ed.). Li Livres de jostice et de plet. Paris, Firmin Didot, 1850. 
En el siglo XIV el jurista italiano Cino da Pistoia ahonda en la condena penal del lesbianismo con la publicación en el año 1314 de su obra Lectura in Codicem $^{27}$, iniciativa legislativa que reinterpreta el derecho romano recogido en el Código de Justianiano. Da Pistoia parte de la Lex Foedissima, promulgada por Diocleciano y Maximiano en el año 287 a.C. que condenaba la prostitución y el libertinaje, y la amplia para englobar en sus implicaciones penales a las mujeres acusadas de mantener relaciones lésbicas. Ya en el siglo XV el jurista italiano Bartolomeo de Saliceto retoma esta interpretación de la Lex Foedissima para condenar el lesbianismo con la pena de muerte en el año 1400. La interpretación de Bartolomeo de Saliceto del derecho romano se convierte en un referente condenatorio del lesbianismo en Europa occidental hasta el siglo XVIII ${ }^{28}$.

No obstante, pese al interés legislativo que despierta el lesbianismo hay muy pocas referencias concretas de la aplicación de estas leyes y no se percibe que existiera una persecución generalizada, que sí parece más exhaustiva en el caso de la homosexualidad masculina. La escasez de procedimientos penales contra mujeres acusadas de mantener relaciones lésbicas indica que la visibilidad social de tales relaciones debió ser muy limitada. Por el contrario, las referencias a actitudes sodomitas entre los hombres son muy habituales debido, en parte, a que tales acusaciones vertidas sobre eclesiásticos motivaron un recrudecimiento de la normativa patrística para paliar tales actitudes en el seno de la Iglesia. Además, la frecuente confusión terminológica entre los conceptos de sodomía y herejía en los autos inquisitoriales subraya que podían existir motivaciones políticas en tales acusaciones como se evidencia en la acusación de sodomía aplicada al conjunto de la Orden del Temple en el siglo XIV.

En diez siglos sólo existen una docena de alusiones al lesbianismo siempre ligadas a la condena eclesiástica, la herejía y, especialmente a la brujería ya que el lesbianismo constituye una acusación habitual en los casos de brujería. La asociación entre el lesbianismo y la brujería responde fundamentalmente a la percepción social de las brujas que conformaba el imaginario social de la Baja Edad Media ${ }^{29}$. La mayoría de los tratados demonológicos señala a las brujas como mujeres que condensan en sí mismas todos los pecados y

27. Cino, Da Pistoia. Lecura in Codicem. Torresanus de Asula, 1493.

28. Crompton, Louis. Homosexuality \& Civilization. Cambridge, Belknap, 2006.

29. BETETA, Yolanda. «Los delitos de las brujas. La pugna por el control del cuerpo y los saberes femeninos» en Cristina Segura \& Isabel del Val Valdivieso (eds.). La participación de las mujeres en lo político. Mediación, representación y toma de decisiones. Madrid, Almudayna, 2011. Pp. 293-322. 
desviaciones derivadas de la caída edénica, entre los que destaca la lascivia y la insaciabilidad sexual ${ }^{30}$; dos debilidades que determinan el gusto de las brujas por las relaciones lésbicas y el bestialismo (relaciones sexuales con machos cabríos).

Paradójicamente, las monjas constituyen junto a las brujas el segundo referente de supuestas acusaciones lésbicas. Los Concilios de París (1212) y de Ruan (1214) les prohibieron dormir juntas y les obligaron a mantener sus cuartos cerrados e iluminados durante la noche. Las suspicacias sobre las conductas de las religiosas en los conventos constituyen una constante en el imaginario androcéntrico medieval fundamentalmente desde los siglos XII y XIII. La proyección pública de las beguinas y beatas, que por su condición de mujeres solteras y seglares, desafiaban la tradicional jerarquía eclesiástica, y la influencia de las místicas, cuyas revelaciones cuestionaban la supuesta incapacidad femenina para interpretar las Sagradas Escrituras, difundieron nuevas formas de religiosidad en las cuales las mujeres adoptan un papel activo al margen de los cánones androcéntricos. Este desafío femenino a las normativas patriarcales evidenció la capacidad de las mujeres para reivindicar una forma de vivir y sentir la religión alejada de la tradicional misoginia patrística.

En base a los desafíos que plantean las nuevas formas de espiritualidad femenina se articula una red política, social e ideológica con el objetivo de coartar cualquier intento de liberación femenina en el seno de la religiosidad cristiana. La política reformista del Cardenal Cisneros constituye el punto final de la deslegitimación de la religiosidad de las beguinas y beatas, al forzar su integración en órdenes religiosas y frenar el desarrollo de los monasterios de mujeres como centros de cultura abiertos a la sociedad. Es el temor a lo desconocido, a lo que ocultan los muros de los conventos, el miedo al poder castrador de las mujeres, lo que emerge tras las críticas androcéntricas sobre las supuestas debilidades de las monjas. Unas debilidades entre las que se incluyen la lascivia, la sodomía, la carnalidad, la heterodoxia y los pactos diabólicos, como señalan numerosos autores bajomedievales.

Un ejemplo de la asociación que la mentalidad patriarcal bajomedieval estableció entre las religiosas, la lascivia y la heterodoxia es la obra Diálogo de mujeres de Cristóbal de Castillejo ${ }^{31}$. El autor dedica un capítulo de la obra a

30. BETETA, Yolanda. Súcubos, hechiceras y monstruos femeninos. Estrategias de desautorización femenina en la ficción bajomedieval. Madrid, Almudayna, 2011.

31. BETETA, Yolanda. «Canon androcéntrico y religiosidad femenina. Sobre los pecados monjiles en el Diálogo de Mujeres de Cristóbal de Castillejo» en Cristina Segura (ed.). La Querella de las Mujeres X. Algunos textos relacionados con la Querella. Madrid, Almudayna, 2011, pp. 43-76. 
condenar las «enbidias, odios, coxquillas, differeçias y rrenzillas y corages y quistiones y barajas» de las religiosas (vv. 1500-1630). Señala que las monjas carecen de la espiritualidad necesaria en la práctica de los sacramentos (vv. 1520-1530) debido a que la naturaleza pecaminosa de las mujeres inhabilita su capacidad de arrepentimiento (vv. 1665-ss). Además, considera que esta falta de espiritualidad constituye la base de las conductas sacrílegas, que desvirtúan el culto apostólico y convierten a Magdalena en un igual frente a los evangelistas (vv. 1611-1614), y condena los supuestos casos de religiosas que abandonan los conventos para dedicarse a la prostitución tentadas por «el gusano venenoso de Cupido» (vv. 1780-1950).

Tras la crisis del sistema feudal y la aparición de los Estados modernos se sucede una renovación espiritual e ideológica que convierte el lesbianismo en un fenómeno de interés creciente sobre todo en los círculos burgueses y cortesanos. Uno de los episodios más conocidos de sentencias inquisitoriales contra mujeres acusadas de mantener relaciones lésbicas es el caso de la religiosa Benedetta Carlini cuya historia sacó a la luz la historiadora Judith C. Brown $^{32}$. A finales del siglo XVII Benedetta Carlini era abadesa del convento de la Madre de Dios en Pescia (Italia) donde según sus propias declaraciones experimentó diversas experiencias místicas. La jerarquía eclesiástica, imbuida del espíritu contrarreformista, silenció el misticismo de Benedetta bajo la acusación inquisitorial de herejía y sodomía. El proceso se basó en las declaraciones de la hermana Bartolomea que alegó las presiones a las que le sometió Benedetta para mantener relaciones sexuales tras las que experimentaban epifanías místicas. La confesión de Bartolemea fue suficiente para que Benedetta fuera despojada de su posición de abadesa y condenada a reclusión hasta su muerte acaecida treinta y cinco años después. El Tratado de enfermedades crónicas $^{33}$ de Soranos señala que la causa de tal sentencia se debe a que Benedetta «actuaba como si fuera un hombre, ella se movía encima de la subordinada con tal intensidad que ambas quedaban corrompida».

El caso de Sor Benedetta Carlini despertó el interés de algunos literatos de los siglos XVII y XVIII, que sitúan el lesbianismo como tema central de varias novelas y poemas. A finales del siglo XVI el escritor francés Pierre de Bourdeille, señor de Brântome, admitía en su obra Vida de las damas galan$t^{2} s^{34}$ que las relaciones sexuales entre mujeres se habían convertido en una

32. Brown, Judith C. Immodest Acts: The Life of a Lesbian Nun in Renaissance Italy. New York, Oxford University Press, 1986.

33. Soranos, M. Tratado de enfermedades crónicas. Madrid, Rivadeneyra, 1832.

34. Bourdeille, Pierre de. Vida de las damas galantes. Traducción de Tomás Cambó. Madrid, Aguilar, 1925. 
moda trasladada de Italia a la corte francesa por una dama noble a quien no nombra, pero por sus descripciones se deduce que hace referencia a Catalina de Medici, reina de Francia. En opinión del señor de Brântome la reina habría sido el ejemplo de un grupo de mujeres conocidas con el sobrenombre de «batallón volante» cuyo rasgo definitorio era la preferencia por «hacer el amor entre ellas» antes que quedar embarazadas y perder su honorabilidad. El autor señala que estas inclinaciones sexuales se dan entre mujeres de diversos estamentos socioeconómicos, incluyendo, por tanto, desde la alta aristocracia hasta el campesinado. En referencia a este último estamento, Pierre de Bourdeille destaca un caso del que tuvo constancia en uno de sus frecuentes viajes a Italia donde documenta la existencia de mujeres casadas entre sí. Relata el caso de una joven llamada María que fue acusada y condenada a la horca por vestirse de hombre y contraer matrimonio con otra mujer.

Es Pierre de Bourdeille quien utiliza por primera vez el término lesbiana para aludir a las mujeres que mantienen relaciones entre ellas en alusión a la isla de Lesbos, hogar de la poetisa Safo. No obstante, aunque el término se acuña en el siglo XVI se registran otras denominaciones anteriores para denominar a aquellas mujeres que mantenían relaciones lésbicas. Marie Jo Bonnet subraya la evolución terminológica del lesbianismo ${ }^{35}$. Durante la Edad Media las relaciones lésbicas no se designaban conceptualmente y en general se extrapolaba el término sodomía que etimológicamente señala las relaciones homosexuales masculinas. Es en el Renacimiento cuando surgen diversos términos para referirse a las relaciones lésbicas siendo los más conocidos los conceptos tríbade, fricatriz y lesbiana. Destaco especialmente el término «tríbade» debido a sus implicaciones con la fisiología y sexualidad femenina.

El término «tríbade» proviene del latín y su significado es «frotar o frotarse». Con este término el lesbianismo se define a través de una actividad sexual que, en el imaginario androcéntrico, es opuesta a la naturaleza de la sexualidad heterosexual. El carácter fálico de la heterosexualidad se opone, por tanto, al supuesto carácter asexual del lesbianismo, que carece de la capacidad de penetración. La significación del término «tríbade» señala la trascendencia de las ideas androcéntricas sobre la sexualidad y la fisiología femenina en la percepción social del lesbianismo.

La mentalidad patriarcal de la Europa moderna consideraba que la vagina era una versión cóncava del pene por lo que la sexualidad femenina se perfilaba en torno a la idea de «imperfección». El cuerpo masculino se proyectaba

35. BONNET, Marie-Joe. Les Relations amoureuses entre les femmes du XVI e au XX e siècle. Essai historique, Éditions Odile Jacob, 1995. 
como el paradigma de la perfección según el discurso patrístico por lo que la ausencia del pene en las mujeres se interpretaba en términos de imperfección. La visión de la naturaleza femenina como un fenómeno imperfecto en virtud de la caída edénica (la expiación del pecado original recae en las mujeres) sienta las bases ideológicas sobre las que se edifican los prejuicios en torno a los casos de lesbianismo y hermafroditismo.

La literatura médica moderna estableció una correlación unívoca entre el clítoris y el lesbianismo. El tratado médico Examen de ingenios para las ciencias de Juan Huarte de San Juan, que tuvo una amplia difusión en Europa subrayaba la creencia de que las mujeres que poseían un clítoris prominente tenían la facultad de penetrar a otras mujeres. Partiendo de esta tesis, el lesbianismo se concibe como una relación entre mujeres en la que de alguna manera se intenta reproducir la penetración inherente a las relaciones heterosexuales según el imaginario androcéntrico. Por lo tanto, el término tríbade (literalmente «frotar o frotarse») guarda relación con la percepción del clítoris como un pene imperfecto, que además provoca un deseo femenino incontrolable ${ }^{36}$; una percepción que motiva que durante siglos la masturbación femenina y el sexo lésbico fueran sinónimos ${ }^{37}$.

En el siglo XVII las diferencias de clase se convirtieron en un factor determinante en las prácticas lésbicas. La mentalidad patriarcal definió a las tríbades como mujeres pertenecientes a las clases socioeconómicas menos privilegiadas cuya finalidad era arrastrar a las mujeres virtuosas al libertinaje. La sátira literaria y la retórica comenzaron además a emplear el lesbianismo como un arma arrojadiza en la praxis política. Por ejemplo, en Inglaterra se difundieron rumores de que la reina Ana I de Gran Bretaña mantenía un apasionado romance con Sarah Churchill, que se convirtió en duquesa de Marlborough gracias a su proximidad con la reina. Cuando la duquesa fue desbancada como la favorita de la reina, la misma Churchill esparció rumores de que la reina tenía relaciones con sus damas de compañía. También hubo rumores sobre María Antonieta durante algunos meses entre 1795 y 1796 y sobre la reina María Carolina, hermana de la anterior y esposa del rey Fernando I de las Dos Sicilias, de la que se decía que era amante de Lady Emma Lyon.

A partir del siglo XVI comienzan a surgir voces femeninas que redirigen la atención de los/as lectores/as hacia la sexualidad femenina, incluyendo, entre otras relaciones afectivas, las relaciones amorosas entre mujeres. La mayoría de las obras ensalza la vertiente amorosa, idílica y romántica del amor entre

36. AlDRICH, Robert. Gay Life and Culture: A World History, Thames \& Hudson, Ltd, 2006. 37. Jennings, Rebecca. A Lesbian History of Britain, Greenwood World Publishing, 2007.

Feminismo/s 19, junio 2012, pp. 29-49 
mujeres que, en muchas ocasiones, se opone a las relaciones afectivas heterosexuales marcadas por los matrimonios pactados y los intereses familiares. El listado de autoras que escriben sobre el amor femenino y sobre las mujeres que experimentan relaciones lésbicas es amplio y diverso a partir de los siglos modernos y allanan el camino hacia la construcción de las identidades lésbicas que se forjan y visibilizan públicamente a partir del siglo XIX. Es el caso de Louise Labé ${ }^{38}$ y sus obras literarias dedicadas a su amiga Clémence de Bourges, las obras de Madeleine de Scudéry ${ }^{39}$ protagonizadas por una nueva Safo que revaloriza una nueva visión de la amistad femenina y rechaza el matrimonio en un convulso contexto político, social e ideológico determinado por La Fronda $^{40}$, las actividades de Madame d'Aiguillon ${ }^{41}$ y Madame du Vigean ${ }^{42}$, que mostraron públicamente su inclinación por la sexualidad lésbica o Madame de Murat $^{43}$, que durante el reinado de Luis XIV fue encarcelada por orden

38. Louise Labé (Lyon, 1525-1566). Poetisa francesa perteneciente a la Escuela Lionesa del Renacimiento. Conocida bajo los sobrenombres de la «Safo de Lyon»o «la Ninfa del Ródano». Escribe obras en prosa y en verso. En este último estilo, publica tres Elegías al estilo de las Heroínas de Ovidio y veinticuatro sonetos muy conocidos que narran la pasión de una mujer en los moldes del Neoplatonismo y el Petrarquismo y que destacan por su eroticidad. En prosa escribe un prefacio feminista a sus propias obras en el que urge a las mujeres a escribir y que está dedicado al joven noble lionés Clemence de Bourges. También escribe una alegoría dramática titulada Debat de Folie et d'Amour (Debate entre la Locura y el Amor), inspirada en el Elogio de la locura de Erasmo que inspiró una de las fábulas de Jean de la Fontaine.

39. Madeleine de Scudéry (París, 1607-1701). Escritora francesa que publica bajo el pseudónimo de Safo y crea el Salón Literario más destacados del Preciosismo. Escribió voluminosas novelas desprovistas de toda semblanza histórica pero en las que se podían reconocer fácilmente los retratos de personajes históricos trasladando a la Antigüedad los valores propios de la sociedad francesa del XVII: Ibrahim ou l'Illustre Bassa (4 volúmenes, 1642); Arteméne ou le Grand Cyrus (10 volúmenes, 1649-1653); Clélie, histoire romaine (10 volúmenes, 1654-1660); Almahide ou l'esclave reine (8 volúmenes, 1660); Matilde d'Aguilar, histoire espagnole (1667).

40. Madeleine de Scudéry puso de manifiesto en Artaméne ou le grand Cyrus su aversión hacia el matrimonio a través de las reflexiones de su heroína Safo, que declaraba que esta institución era una tiranía. Esta novela fue considerada por algunos críticos literarios como la primera novela moderna en la que, sin hacer apología de la sedición política, dejó entrever las simpatías que sentía por los Frondeses. El personaje de Safo constituye el primer aviso de la toma de conciencia acerca del hecho de que, tras la Fronda, las mujeres quedarían relegadas no pudiendo poner de manifiesto sus conocimientos y su talento más que en la esfera privada.

41. Marie-Madeleine de Vignerod (1604-1675). Sobrina del cardenal Richelieu. Lideró un Salón Literario y financió la creación del convento de las Ursulinas de Quebec y el convento de la Congregación de Notre-Dame de Montreal.

42. Anne du Vigean, duquesa de Richelieu.

43. Henriette-Julie de Castelnau (Brest, 1670-1716). Escritora francesa conocida sobre todo por sus cuentos de hadas que marcaron una tendencia literaria en la Europa del

Feminismo/s 19, junio 2012, pp. 29-49 
del rey bajo la acusación de amar a mujeres, dilapidar la fortuna familiar y tener una moral disoluta. Ya en el siglo XVIII destaca la presencia de Frances Brudenell, duquesa de Newburgh, a quien se le atribuye haber liderado un círculo social de tríbadas en Dublín, y la actividad de Therese de Fleury a la que se atribuye la fundación del legendario grupo Anandrynas (1770) integrado exclusivamente por tríbadas. No obstante, es a partir de los siglos XIX y XX donde el lesbianismo comienza a tener mayor visibilidad social.

En líneas generales, el tratamiento que se daba al lesbianismo en las diversas etapas históricas era, dentro de la condena moral y la reprobación social y jurídica, más liviano que el de la homosexualidad masculina, identificada bajo el concepto de sodomía. Se consideraba que la simiente masculina era más importante que la femenina y que por lo tanto cualquier acto sexual no reproductivo constituía una ofensa a Dios. El lesbianismo se consideraba un problema menos grave que las relaciones entre hombres lo que daría lugar a una menor persecución pero también a una mayor ignorancia de la existencia y la identidad de las mujeres lesbianas. Una ignorancia que propició el desarrollo social de lo que en épocas modernas se dio en llamar amor romántico entre mujeres que, bajo la apariencia de una amistad platónica, acogió diversos tipos de relación afectivo-sexual que sobro todo a partir del siglo XVI, facilitó la formación de las identidades lésbicas que aflorarían en los siglos XIX y XX.

\section{Referencias bibliográficas}

AlDRICH, Robert (ed.). Gay Life and Culture: A World History, Thames \& Hudson, 2006.

ALFARACHE, Lorenzo \& Angela G. Identidades lésbicas y cultura feminista: una investigación antropológica. México, D.F. Centro de Investigaciones Interdisciplinarias en Ciencias y Humanidades-Universidad Nacional Autónoma de México, Plaza y Valdés, 2003.

BETETA, Yolanda. Súcubos, hechiceras y monstruos femeninos. Estrategias de desautorización femenina en la ficción bajomedieval. Madrid, Almudayna, 2011.

BETETA, Yolanda. «Los delitos de las brujas. La pugna por el control del cuerpo y los saberes femeninos» en Cristina Segura \& Isabel del Val Valdivieso (eds.). La participación de las mujeres en lo político. Mediación, representación y toma de decisiones. Madrid, Almudayna, 2011. pp. 293-322.

BETETA, Yolanda. «Canon androcéntrico y religiosidad femenina. Sobre los pecados monjiles en el Diálogo de Mujeres de Cristóbal de Castillejo» en Cristina

siglo XVIII y cuya influencia pervive hasta la actualidad. Fue condenada y exiliada a Loches entre los años 1702 y 1709 bajo la acusación de depravación y lesbianismo en un contexto político en el que se cuestiona la política de Luis XIV. 
Segura (ed.). La Querella de las Mujeres X. Algunos textos relacionados con la Querella. Madrid, Almudayna, 2011, pp. 43-76.

BLACKWOOD, Evelyn. «Breaking the mirror: The construction of Lesbianism and the Anthropological Discourse on Homosexuality» en Evelyn Blackwood (ed.): The many faces of Homosexuality: Anthropological approaches to Homosexual Behavior, Harrington Park Press, 1986.

BOnNET, Marie-Joe. Les Relations amoureuses entre les femmes du XVI e au XX e siècle. Essai historique, Éditions Odile Jacob, 1995.

Bremmer, Jan (ed.). From Sappho to de Sade: Moments in the History of Sexuality. Routledge, 1989.

Brown, Judith C. Immodest Acts: The Life of a Lesbian Nun in Renaissance Italy. New York, Oxford University Press, 1986.

Butler, Judith. El grito de Antígona. Barcelona, El Roure, 2001.

BUTLER, Judith. Cuerpos que importan: sobre los límites materiales y discursivos del sexo. Buenos Aires, Paidós, 2005.

Butler, Judith. Deshacer el género. Barcelona, Paidós, 2006.

BUTLER, Judith. Gender trouble: feminism and the subversion of identity, London, Routledge, 2006.

CAnAdé, Francesca \& SHEIngom, Pamela. Same sex love and desire among women in the Middle Ages. New York, Palgrave, 2001.

Cavin, Susan. Lesbian origins. San Francisco, Ism Press, 1989.

Clarke, Cherryl. Lesbianism: an act of resistance. London, Persephone Press, 1981

Crompton, Louis. Homosexuality E Civilization. Cambridge, Belknap, 2006.

DE JEAn, Joan. Fictions of Sappho, 1546-1937. Chicago, University of Chicago Press, 1989.

FADERMAN, Lillian. Surpassing the love of men: romantic friendship and love between women from the Renaissance to the present. London, The women's press, 1991.

GAY, Judith.Mummies and babies and friends and lovers in Lesotho. Harrington Park Press, 1986.

Gimeno, Beatriz. Historia y análisis político del lesbianismo: la liberación de una generación. Barcelona, Gedisa editorial, 2005.

Halberstam, Judith. Masculinidad femenina. Barcelona, Egales, D.L. 2008.

JEFFREYS, Sheila. La herejía lesbiana: una perspectiva feminista de la revolución sexual lesbiana. Madrid, Cátedra, D.L. 1996.

JENNINGS, Rebecca. A Lesbian History of Britain, Greenwood World Publishing, 2007.

Kitzinger, Celia. The social construction of lesbianism. London, Sage, 1990.

LAGARDE, Marcela. Género y feminismo: derechos humanos y democracia. Madrid, Horas y Horas, 2000.

RICH, Adrianne. "Compulsory Heterosexuality and lesbian existence» en Signs 5 (4), 1980, pp. 631-660. 
SÁnCHEZ, Carmen. Arte y erotismo en el mundo clásico. Madrid, Siruela 2005.

SANKAR, Andrea. «Sisters and brothers, lovers and enemies» en Evelyn Blackwood (ed.): The many faces of Homosexuality: Anthropological approaches to Homosexual Behavior, Harrington Park Press, 1986.

VIÑuALES, Olga. Identidades lésbicas: discursos y prácticas. Barcelona, Bellaterra, 2000.

WIERINGA, Saskia.»Feminist Anthropology since the Mid-Seventies: From Monocausality to Diversity, a personal view», en M. Krueger (ed.): Was Heisst Hier Eigentlich Feministisch?, Frankfurt, Donat, 1993.

ZITA, Jacqueline. «Historical amnesia and the lesbian continuum», en Signs: Journal of women in culture and society, 7 (1), (1981), pp. 172-187. 